\title{
Ichneumonidae (Hymenoptera) species new to the fauna of Norway
}

\author{
Andrei E. Humala ${ }^{\dagger, \ddagger}$, Alexey Reshchikov $§, \dagger$ \\ † University of Oslo, Natural History Museum, Department of Research and Collections, Oslo, Norway \\ $\ddagger$ Forest Research Institute KRC RAS, Petrozavodsk, Russia \\ $\S$ Swedish Museum of Natural History, Stockholm, Sweden
}

Corresponding author: Andrei E. Humala (humala@krc.karelia.ru)

Academic editor: Jose Fernandez-Triana

Received: 01 Jan 2014 | Accepted: 18 Feb 2014 | Published: 25 Feb 2014

Citation: Humala A, Reshchikov A (2014) Ichneumonidae (Hymenoptera) species new to the fauna of Norway. Biodiversity Data Journal 2: e1047. doi: 10.3897/BDJ.2.e1047

\section{Abstract}

The present paper contains new distributional records for 61 species of ichneumon wasps (Hymenoptera, Ichneumonidae) previously unknown for Norway, six of them are reported from Scandinavia for the first time.

\section{Keywords}

Ichneumon wasps, Ichneumonidae, Norway, Scandinavia, new records

\section{Introduction}

The Ichneumonidae are the largest family within Hymenoptera, and one of the largest among insects. Due to the great diversity and difficulties in identification of many species in this group, our knowledge of the Norwegian fauna remains insufficient. During the last twenty years, more than 500 species of Ichneumonidae were reported as new to the fauna of Norway (Kopelke 1994, Riedel and Berg 1997, Riedel et al. 2000, Horstmann 2004, Riedel et al. 2005, Riedel and Hansen 2007, Kasparyan and Kopelke 2009, Hansen et al. 2010, Kasparyan and Kopelke 2010, Humala and Reshchikov 2012, Riedel and Hansen 
2012, Reshchikov 2013, Riedel and Hansen 2013). Although in total more than 1700 species of Ichneumonidae have been listed for Norway so far, the published checklists from adjacent territories (Koponen et al. 1995, Broad 2011) suggest that the Norwegian list is still very incomplete.

\section{Material and methods}

During the 2011 field season, ichneumonids were sampled in many localities across the southern half of the country. The division of Norway in administrative regions (counties) applied in faunistic studies is shown on the map (Fig. 1). The northern-most provinces of Norway were not sampled due to time constraints of the project. The light-weight Malaise traps were the main method of collecting, although yellow pan traps and netting were also used.

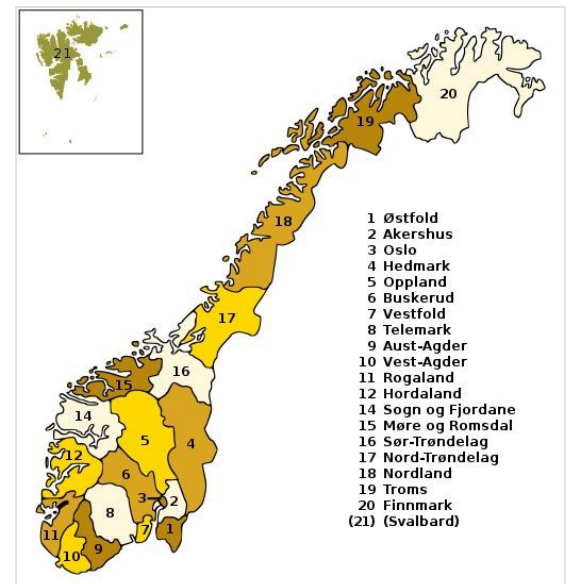

Figure 1.

The division of Norway in administrative regions (counties).

The original collected material and voucher specimens for DNA barcoding are deposited at the Natural History Museum, University of Oslo, Norway.

Additional material was borrowed from the following museums: The Museum of Natural History and Archaeology, Trondheim; University Museum of Bergen; reared material of the genus Saotis Förster, 1869 was provided by Department of Biology, University of Eastern Finland. Species identification in subfamily Ctenopelmatinae was confirmed by comparison with the type specimens deposited in Swedish museums (The Swedish Museum of Natural History, Stockholm; Zoological Institute of Uppsala University; Zoological Institute, Lund). All taxa are listed in alphabetic order according to the recent Catalogue of World Ichneumonidae (Yu et al. 2012). Species distributions are based on the same catalogue and Fauna Europaea website (Achterberg van and Zwakhals 2004) with minor changes. The terms Palaearctic and Western Palaearctic are used to specify how wide is 
distribution. Species distributions in Fennoscandia, in the countries adjacent or faunistically similar to Norway are given in more detail after biogeographic region name. The following abbreviations are used in the text:

NTNU - Trondheim Museum of Natural History and Archaeology (Vitenskapsmuseet)

NW - North-Western

UEF - University of Eastern Finland

ZMUN - Natural History Museum, University of Oslo

\section{Species new to Norway}

Subfamily Acaenitinae Förster, 1869

Tribe Coleocentrini Clément, 1938

\section{Coleocentrus caligatus Gravenhorst, 1829}

Material

a. order: Hymenoptera; family: Ichneumonidae; genus: Coleocentrus; specificEpithet: caligatus; scientificNameAuthorship: Gravenhorst, 1829; country: Norway; stateProvince: Aust-Agder; verbatimLocality: Risør; eventDate: 28.VI.1906; individualCount: 1; sex: female; recordedBy: Thorstensen; identifiedBy: Andrei E. Humala; collectionCode: ZMUN

Distribution: Palaearctic; Finland, Sweden and NW Russia (Karelia).

Subfamily Adelognathinae Thomson, 1888

\section{Adelognathus difformis Holmgren, 1857}

Material

a. order: Hymenoptera; family: Ichneumonidae; genus: Adelognathus; specificEpithet: difformis; scientificNameAuthorship: Holmgren, 1857; country: Norway; stateProvince: Akerhus; verbatimLocality: Ullensaker, Sessvollmoen, Aurtjernet W; verbatimElevation: 200 m; samplingProtocol: Malaise trap; eventDate: 30.VIII-1.X.2008; habitat: sandy pine forest; individualCount: 1; sex: female; recordedBy: L.O. Hansen; identifiedBy: Andrei E. Humala; institutionCode: ZMUN

Distribution: Holarctic; Sweden, Finland and NW Russia (Leningrad region). 


\section{Adelognathus rufithorax Kasparyan, 1990}

\section{Material}

a. order: Hymenoptera; family: Ichneumonidae; genus: Adelognathus; specificEpithet: rufithorax; scientificNameAuthorship: Kasparyan, 1990; country: Norway; stateProvince: Sogn og Fjordane; verbatimLocality: Leikanger, Kvinnefossen waterfall; verbatimElevation: $60 \mathrm{~m}$; verbatimLatitude: $61^{\circ} 12^{\prime} 30.2^{\prime \prime} \mathrm{N}$; verbatimLongitude: $06^{\circ}$ 38'52.0" E; samplingProtocol: Malaise trap; eventDate: 6.VII-30.VIII.2011; habitat: spruce forest with juniper; individualCount: 1; sex: female; recordedBy: Andrei E. Humala \& Alexey Reshchikov; identifiedBy: Andrei E. Humala; institutionCode: ZMUN

Distribution: Palaearctic; NW Russia (Leningrad region).

Notes: New to Scandinavia.

\section{Adelognathus stelfoxi Fitton, Gauld \& Shaw, 1982}

\section{Material}

a. order: Hymenoptera; family: Ichneumonidae; genus: Adelognathus; specificEpithet: stelfoxi; scientificNameAuthorship: Fitton, Gauld \& Shaw, 1982; country: Norway; stateProvince: Akerhus; verbatimLocality: Ullensaker, Sessvollmoen W; verbatimElevation: 204 m; samplingProtocol: Malaise trap; eventDate: 26.VI-25.VIII.2007; habitat: sandy pine forest; individualCount: 1; sex: female; recordedBy: L.O. Hansen; identifiedBy: Andrei E. Humala; institutionCode: ZMUN

Distribution: Palaearctic; Finland.

\section{Subfamily Banchinae Wesmael, 1845}

\section{Tribe Glyptini Cushman \& Rohwer, 1920}

\section{Glypta (Glypta) consimilis Holmgren, 1860}

\section{Material}

a. $\quad$ order: Hymenoptera; family: Ichneumonidae; genus: Glypta; subgenus: Glypta; specificEpithet: consimilis; scientificNameAuthorship: Holmgren, 1860; country: Norway; stateProvince: Aust-Agder; verbatimLocality: Lillesand, Froholt; verbatimElevation: 53 m; verbatimLatitude: $58^{\circ} 19^{\prime} 33.9^{\prime \prime} \mathrm{N}$; verbatimLongitude: 08¹8'15.5" E; samplingProtocol: Malaise trap; eventDate: 30.VI-23.VIII.2011; habitat: oak forest; individualCount: 1; sex: female; recordedBy: Andrei E.Humala \& Alexey Reshchikov; identifiedBy: Andrei E. Humala; institutionCode: ZMUN

Distribution: Holarctic; Finland, Sweden, Lithuania and NW Russia (Leningrad region). 


\section{Subfamily Campopleginae Förster, 1869}

\section{Leptocampoplex cremastoides (Holmgren, 1860)}

\section{Materials}

a. order: Hymenoptera; family: Ichneumonidae; genus: Leptocampoplex; specificEpithet: cremastoides; scientificNameAuthorship: (Holmgren, 1860); country: Norway; stateProvince: Aust-Agder; verbatimLocality: Lillesand, Froholt; verbatimElevation: 53 m; verbatimLatitude: 58¹9'33.9" N; verbatimLongitude: 08¹8'15.5" E; samplingProtocol: Malaise trap; eventDate: 30.VI-23.VIII.2011; habitat: oak forest; individualCount: 1; sex: female; recordedBy: Andrei E. Humala \& Alexey Reshchikov; identifiedBy: Andrei E. Humala; institutionCode: ZMUN

b. order: Hymenoptera; family: Ichneumonidae; genus: Leptocampoplex; specificEpithet: cremastoides; scientificNameAuthorship: (Holmgren, 1860); country: Norway; stateProvince: Aust-Agder; verbatimLocality: Lillesand, Vestre Grimevann lake; verbatimLatitude: 58¹9'23.1" N; verbatimLongitude: 08²0'09.1" E; samplingProtocol: Malaise trap; eventDate: 29.VI-23.VIII.2011; habitat: oak forest; individualCount: 1; sex: female; recordedBy: Andrei E. Humala \& Alexey Reshchikov; associatedSequences: voucher for DNA barcoding AH057; identifiedBy: Andrei E. Humala; institutionCode: ZMUN

Distribution: Holarctic; Finland, Sweden and NW Russia (Karelia).

\section{Rhimphoctona (Xylophylax) rufocoxalis (Clément, 1924)}

\section{Materials}

a. order: Hymenoptera; family: Ichneumonidae; genus: Rhimphoctona; subgenus: Xylophylax; specificEpithet: rufocoxalis; scientificNameAuthorship: (Clément, 1924); country: Norway; stateProvince: Nord Trøndelag; verbatimLocality: Høylandet, Skiftesåa; samplingProtocol: Malaise trap; eventDate: 2.VII.1987; individualCount: 1; sex: female; recordedBy: Kaare Aagaard; identifiedBy: Andrei E. Humala; institutionCode: NTNU

b. order: Hymenoptera; family: Ichneumonidae; genus: Rhimphoctona; subgenus: Xylophylax; specificEpithet: rufocoxalis; scientificNameAuthorship: (Clément, 1924); country: Norway; stateProvince: Nord Trøndelag; verbatimLocality: Høylandet, Skiftesåa; samplingProtocol: Malaise trap; eventDate: 22.VII.1987; individualCount: 2; sex: females; recordedBy: Kaare Aagaard; identifiedBy: Andrei E. Humala; institutionCode: NTNU

c. order: Hymenoptera; family: Ichneumonidae; genus: Rhimphoctona; subgenus: Xylophylax; specificEpithet: rufocoxalis; scientificNameAuthorship: (Clément, 1924); country: Norway; stateProvince: Nord Trøndelag; verbatimLocality: Høylandet, Tverrråa; verbatimElevation: 340 m; samplingProtocol: Malaise trap; eventDate: 30.VII.1986; individualCount: 1; sex: female; recordedBy: Kaare Aagaard; identifiedBy: Andrei E. Humala; institutionCode: NTNU

Distribution: Palaearctic; Sweden and NW Russia (Karelia). 


\section{Subfamily Cryptinae Kirby, 1837}

\section{Tribe Cryptini Kirby, 1837}

\section{Ateleute linearis Förster, 1871}

\section{Material}

a. order: Hymenoptera; family: Ichneumonidae; genus: Ateleute; specificEpithet: linearis; scientificNameAuthorship: Förster, 1871; country: Norway; stateProvince: Aust-Agder; verbatimLocality: Lillesand, Vestre Grimevann lake; verbatimLatitude: 58¹9'23.1" N; verbatimLongitude: $08^{\circ} 20^{\prime} 09.1$ " E; samplingProtocol: Malaise trap; eventDate: 29.VI-23.VIII.2011; habitat: oak forest; individualCount: 1; sex: male; recordedBy: Andrei E. Humala \& Alexey Reshchikov; associatedSequences: voucher for DNA barcoding AH056; identifiedBy: Andrei E. Humala; institutionCode: ZMUN

Distribution: Western Palaearctic; Finland, Sweden and NW Russia (Karelia, Leningrad region).

\section{Subfamily Ctenopelmatinae Förster, 1869}

\section{Tribe Ctenopelmatini Förster, 1869}

\section{Ctenopelma boreale Holmgren, 1857}

\section{Material}

a. order: Hymenoptera; family: Ichneumonidae; genus: Ctenopelma; specificEpithet: boreale ; scientificNameAuthorship: Holmgren, 1857; country: Norway; stateProvince: Hedmark; verbatimLocality: Vestmarka; eventDate: 5. VII.1997; individualCount: 1; sex: male; recordedBy: Ole Lønnve; identifiedBy: Alexey Reshchikov; institutionCode: ZMUN

Distribution: Palaearctic; Sweden, Finland and NW Russia (Karelia).

Notes: According to Kasparyan (Kasparyan and Khalaim 2007) C. boreale Holmgren is a distinct species.

\section{Ctenopelma lapponicum Holmgren, 1857}

\section{Material}

a. order: Hymenoptera; family: Ichneumonidae; genus: Ctenopelma; specificEpithet: lapponicum; scientificNameAuthorship: Holmgren, 1857; country: Norway; stateProvince: Oppland; verbatimLocality: Dørålsæter; eventDate: 25.VI.1986; individualCount: 1; sex: female; recordedBy: John O. Solem; identifiedBy: Alexey Reshchikov; institutionCode: NTNU

Distribution: Palaearctic; Sweden, Finland and NW Russia (Karelia). 


\section{Ctenopelma nigrum Holmgren, 1857}

\section{Material}

a. order: Hymenoptera; family: Ichneumonidae; genus: Ctenopelma; specificEpithet: nigrum; scientificNameAuthorship: Holmgren, 1857; country: Norway; stateProvince: Vestfold; verbatimLocality: Larvik; eventDate: 25.VI.1911; individualCount: 1; sex: female; recordedBy: Remharat; identifiedBy: Alexey Reshchikov; institutionCode: ZMUN

Distribution: Palaearctic; Sweden, Finland, Latvia and NW Russia (Karelia).

\section{Ctenopelma tomentosum (Desvignes, 1856)}

Material

a. order: Hymenoptera; family: Ichneumonidae; genus: Ctenopelma; specificEpithet: tomentosum; scientificNameAuthorship: (Desvignes, 1856); country: Norway; stateProvince: Østfold; verbatimLocality: Hvaler, Huser; eventDate: 8.VIII.2001; individualCount: 1; sex: female; recordedBy: Ove Sørlibråten; identifiedBy: Alexey Reshchikov; institutionCode: ZMUN

Distribution: Palaearctic; Sweden, Finland, Latvia, Lithuania and NW Russia (Karelia).

\section{Homaspis subalpina Schmiedeknecht, 1913}

\section{Material}

a. order: Hymenoptera; family: Ichneumonidae; genus: Homaspis; specificEpithet: subalpina ; scientificNameAuthorship: Schmiedeknecht, 1913; country: Norway; stateProvince: Hedmark; verbatimLocality: Kongsvinger, Vennerberg, Langerutberget; eventDate: 3.VI.2005; individualCount: 1; sex: female; recordedBy: Ove Sørlibråten; identifiedBy: Alexey Reshchikov; institutionCode: ZMUN

Distribution: Palaearctic; Finland and NW Russia (Karelia).

\section{Xenoschesis (Polycinetis) fulvicornis (Kriechbaumer, 1891)}

\section{Material}

a. order: Hymenoptera; family: Ichneumonidae; genus: Xenochesis; subgenus: Polycinetis; specificEpithet: fulvicornis; scientificNameAuthorship: (Kriechbaumer, 1891); country: Norway; stateProvince: Hedmark; verbatimLocality: Eidskog, Vestmarka, Hornåsen; eventDate: 13.VI.2004; individualCount: 1; sex: female; recordedBy: Ove Sørlibråten; identifiedBy: Alexey Reshchikov; institutionCode: ZMUN

Distribution: Western Palaearctic; Finland and Latvia. 
Tribe Euryproctini Thomson, 1883

\section{Euryproctus geniculosus (Gravenhorst, 1829)}

\section{Material}

a. order: Hymenoptera; family: Ichneumonidae; genus: Euryproctus; specificEpithet: geniculosus; scientificNameAuthorship: (Gravenhorst, 1829); country: Norway; stateProvince: Aust-Agder; verbatimLocality: Arendal, Hissey; eventDate: 18.VII.1954; individualCount: 1; sex: female; recordedBy: A. Bakke; identifiedBy: Alexey Reshchikov; institutionCode: ZMUN

Distribution: Western Palaearctic; Denmark, Finland, Sweden, Latvia, Lithuania and NW Russia (Leningrad region).

\section{Syndipnus pannicularius (Holmgren, 1857)}

\section{Material}

a. order: Hymenoptera; family: Ichneumonidae; genus: Syndipnus; specificEpithet: pannicularius; scientificNameAuthorship: (Holmgren, 1857); country: Norway; stateProvince: Hedmark; verbatimLocality: Storelvdal, Atnaelv, Solbakken; samplingProtocol: Malaise trap; eventDate: 24.VI.1986; individualCount: 1; sex: female; recordedBy: John O. Solem; identifiedBy: Alexey Reshchikov; institutionCode: NTNU

Distribution: Holarctic; Sweden and Finland.

\section{Tribe Mesoleiini Thomson, 1883}

\section{Saotis albionis Kasparyan, 2007}

\section{Material}

a. order: Hymenoptera; family: Ichneumonidae; genus: Saotis; specificEpithet: albionis; scientificNameAuthorship: Kasparyan, 2007; country: Norway; stateProvince: Hedmark; verbatimLocality: Atnaelv, Solbakken; samplingProtocol: Malaise trap; eventDate: 24.VI.1986; individualCount: 2; sex: females; recordedBy: John O. Solem; identifiedBy: Alexey Reshchikov; institutionCode: NTNU

Distribution: Holarctic; United Kingdom, Finland.

\section{Saotis heteropus (Thomson, 1883)}

\section{Material}

a. order: Hymenoptera; family: Ichneumonidae; genus: Saotis; specificEpithet: heteropus; scientificNameAuthorship: (Thomson, 1883); country: Norway; stateProvince: Hedmark; verbatimLocality: Atnaelv, Solbakken; samplingProtocol: Malaise trap; eventDate: 24.VI.1986; individualCount: 1; sex: female; recordedBy: John O. Solem; identifiedBy: Alexey Reshchikov; institutionCode: NTNU 
Distribution: Holarctic; Sweden, Finland and NW Russia (Leningrad region).

Saotis pygidiator subsp. arctor Kasparyan, 2009

Material

a. order: Hymenoptera; family: Ichneumonidae; genus: Saotis; specificEpithet: pygidiator; infraspecificEpithet: arctor, scientificNameAuthorship: Kasparyan, 2009; country: Norway; stateProvince: Nordland; verbatimLocality: Bodø, Nordstranda, Skjelstadt; eventDate: 27.VI.1995; individualCount: 1; sex: female; recordedBy: H. Elven; identifiedBy: Alexey Reshchikov; institutionCode: UEF

Distribution: Western Palaearctic.

Notes: New to Scandinavia.

\section{Tribe Perilissini Thomson, 1883}

\section{Perilissus compressus Thomson, 1883}

\section{Material}

a. order: Hymenoptera; family: Ichneumonidae; genus: Perilissus; specificEpithet: compressus; scientificNameAuthorship: Thomson, 1883; country: Norway; stateProvince: Hedmark; verbatimLocality: Storelvdal, Atnaelv, Solbakken; samplingProtocol: Malaise trap; eventDate: 24.VI.1986; individualCount: 1; sex: female; recordedBy: John O. Solem; identifiedBy: Alexey Reshchikov; institutionCode: NTNU

Distribution: Western Palaearctic; Sweden and Finland.

\section{Synoecetes anterior (Thomson, 1894)}

Material

a. $\quad$ order: Hymenoptera; family: Ichneumonidae; country: Norway; stateProvince: Hedmark; verbatimLocality: Storelvdal, Atnaelv, Solbakken; samplingProtocol: Malaise trap; eventDate: 24.VI.1986; individualCount: 1; sex: female; recordedBy: John O. Solem; identifiedBy: Alexey Reshchikov; institutionCode: NTNU

Distribution: Western Palaearctic; Sweden. 


\section{Tribe Pionini Smith \& Shenefelt, 1955}

\section{Asthenara socia (Holmgren, 1857)}

\section{Material}

a. order: Hymenoptera; family: Ichneumonidae; genus: Asthenara; specificEpithet: socia; scientificNameAuthorship: (Holmgren, 1857); country: Norway; stateProvince: NordTrøndelag; verbatimLocality: Hoylandet, Tverråa stream; verbatimElevation: 340 m; verbatimLatitude: $64.39^{\circ} \mathrm{N}$; verbatimLongitude: $12.08^{\circ} \mathrm{E}$; eventDate: 18.VII.1986; individualCount: 8; sex: 7 females, 1 male; recordedBy: K. Aagaard; identifiedBy: Alexey Reshchikov; institutionCode: NTNU

Distribution: Western Palaearctic; Sweden, Finland, Lithuania and NW Russia (Karelia).

\section{Glyptorhaestus punctatus (Thomson, 1890)}

\section{Material}

a. order: Hymenoptera; family: Ichneumonidae; genus: Glyptorhaestus; specificEpithet: punctatus; scientificNameAuthorship: (Thomson, 1890); country: Norway; stateProvince: Akerhus; verbatimLocality: Oslo, Ekebergsraninger; samplingProtocol: Malaise trap; eventDate: 29.V-27.VI.2007; individualCount: 1; sex: female; recordedBy: L.O. Hansen; identifiedBy: Alexey Reshchikov; institutionCode: ZMUN

Distribution: Palaearctic; Sweden, Finland, Lithuania and Latvia.

\section{Lethades lapponator Hinz, 1976}

\section{Material}

a. order: Hymenoptera; family: Ichneumonidae; genus: Lethades; specificEpithet: Iapponator ; scientificNameAuthorship: Hinz, 1976; country: Norway; stateProvince: Sør-Trøndelag; verbatimLocality: Oppdal, Kongsvoll, Gavalibk; eventDate: 13.VII.1982; individualCount: 4; sex: females; recordedBy: John O. Solem; identifiedBy: Alexey Reshchikov; institutionCode: NTNU

Distribution: Western Palaerctic; Sweden.

\section{Rhaestus ophthalmicus (Holmgren, 1857)}

\section{Material}

a. order: Hymenoptera; family: Ichneumonidae; genus: Rhaestus; specificEpithet: ophthalmicus; scientificNameAuthorship: (Holmgren, 1857); country: Norway; stateProvince: Sør-Trøndelag; verbatimLocality: Oppdal, Kongsvoll, Blesbekken; samplingProtocol: Malaise trap; eventDate: 10.VII.1980; individualCount: 4; sex: males; recordedBy: John O. Solem; identifiedBy: Alexey Reshchikov; institutionCode: NTNU 
Distribution: Palaearctic; Sweden, Finland and NW Russia (Karelia, Leningrad region).

\section{Sympherta canaliculata (Thomson, 1893)}

\section{Material}

a. order: Hymenoptera; family: Ichneumonidae; genus: Sympherta; specificEpithet: canaliculata; scientificNameAuthorship: (Thomson, 1893); country: Norway; stateProvince: Hedmark; verbatimLocality: Folldal, Atnaelv, Vollen; samplingProtocol: Malaise trap; eventDate: 18-25.VI.1986; individualCount: 1; sex: female; recordedBy: John O. Solem; identifiedBy: Alexey Reshchikov; institutionCode: NTNU

Distribution: Western Palaearctic; Lithuania.

\section{Sympherta jactator (Thunberg, 1824)}

Material

a. order: Hymenoptera; family: Ichneumonidae; genus: Sympherta; specificEpithet: jactator, scientificNameAuthorship: (Thunberg, 1824); country: Norway; stateProvince: Hedmark; verbatimLocality: Storelvdal, Solbakken; samplingProtocol: Malaise trap; eventDate: 24.VIII.1986; individualCount: 13; sex: 3 females, 10 males; recordedBy: John O. Solem; identifiedBy: Alexey Reshchikov; institutionCode: NTNU

Distribution: Western Palaearctic; Sweden and NW Russia (Karelia).

\section{Sympherta splendens (Strobl, 1903)}

\section{Material}

a. order: Hymenoptera; family: Ichneumonidae; genus: Sympherta; specificEpithet: splendens; scientificNameAuthorship: (Strobl, 1903); country: Norway; stateProvince: Akerhus; verbatimLocality: Oppegård, Svartskog, Rødstein; samplingProtocol: Malaise trap; eventDate: 4.VI-4.VII.2004; individualCount: 1; sex: female; recordedBy: Ole Lønnve; identifiedBy: Alexey Reshchikov; institutionCode: ZMUN

Distribution: Palaearctic; Finland and Lithuania.

\section{Sympherta sulcata (Thomson, 1890)}

\section{Material}

a. order: Hymenoptera; family: Ichneumonidae; genus: Sympherta; specificEpithet: sulcata; scientificNameAuthorship: (Thomson, 1890); country: Norway; stateProvince: Akerhus; verbatimLocality: Oppegård, Svartskog, Rødstein; samplingProtocol: Malaise trap; eventDate: 4.VI-4.VII.2004; individualCount: 1; sex: female; recordedBy: Ole Lønnve; identifiedBy: Alexey Reshchikov; institutionCode: ZMUN

Distribution: Palaearctic; Finland, Sweden and NW Russia (Karelia). 


\section{Trematopygus rufator Hinz, 1986}

\section{Material}

a. order: Hymenoptera; family: Ichneumonidae; genus: Trematopygus; specificEpithet: rufator, scientificNameAuthorship: Hinz, 1986; country: Norway; stateProvince: NordTrøndelag; verbatimLocality: Høylandet, Tverråa stream; samplingProtocol: Malaise trap; eventDate: 25.VI.1986; individualCount: 1; sex: female; recordedBy: John O. Solem; identifiedBy: Alexey Reshchikov; institutionCode: NTNU

Distribution: Western Palaearctic; Sweden and Finland.

\section{Trematopygus terebrator Hinz, 1986}

\section{Material}

a. order: Hymenoptera; family: Ichneumonidae; genus: Trematopygus; specificEpithet: terebrator, scientificNameAuthorship: Hinz, 1986; country: Norway; stateProvince: NordTrøndelag; verbatimLocality: Høylandet, Tverråa stream; samplingProtocol: Malaise trap; eventDate: 25.VI.1986; individualCount: 1; sex: female; recordedBy: John O. Solem; identifiedBy: Alexey Reshchikov; institutionCode: NTNU

Distribution: Western Palaearctic; Sweden and Finland.

\section{Subfamily Cylloceriinae Wahl, 1990}

\section{Cylloceria caligata (Gravenhorst, 1829)}

\section{Materials}

a. order: Hymenoptera; family: Ichneumonidae; genus: Cylloceria; specificEpithet: caligata; scientificNameAuthorship: (Gravenhorst, 1829); country: Norway; stateProvince: Hedmark; verbatimLocality: Folldal, Vollen; samplingProtocol: Malaise trap; eventDate: 8.VII.1986; individualCount: 6; sex: males; recordedBy: John O. Solem; identifiedBy: Andrei E. Humala; institutionCode: NTNU

b. order: Hymenoptera; family: Ichneumonidae; genus: Cylloceria; specificEpithet: caligata; scientificNameAuthorship: (Gravenhorst, 1829); country: Norway; stateProvince: Hedmark; verbatimLocality: Folldal, Vollen; samplingProtocol: Malaise trap; eventDate: 23.VIII.1986; individualCount: 2; sex: males; recordedBy: John O. Solem; identifiedBy: Andrei E. Humala; institutionCode: NTNU

c. order: Hymenoptera; family: Ichneumonidae; genus: Cylloceria; specificEpithet: caligata; scientificNameAuthorship: (Gravenhorst, 1829); country: Norway; stateProvince: Hedmark; verbatimLocality: Folldal, Vollen; verbatimElevation: 720 m; verbatimLatitude:

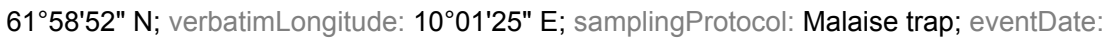
11-18.VIII.2008; individualCount: 1; sex: male; recordedBy: E. Stur; identifiedBy: Andrei E. Humala; institutionCode: NTNU

d. order: Hymenoptera; family: Ichneumonidae; genus: Cylloceria; specificEpithet: caligata; scientificNameAuthorship: (Gravenhorst, 1829); country: Norway; stateProvince: Hedmark; verbatimLocality: Elverum, Starmoen NR (N); samplingProtocol: Malaise trap; eventDate: 29.VII-14.IX.2004; individualCount: 1; sex: female; recordedBy: L.O. Hansen and E. Rindal; identifiedBy: Andrei E. Humala; institutionCode: ZMUN 
e. order: Hymenoptera; family: Ichneumonidae; genus: Cylloceria; specificEpithet: caligata; scientificNameAuthorship: (Gravenhorst, 1829); country: Norway; stateProvince: Akershus; verbatimLocality: Hurdal, Brustad; eventDate: 17.VIII.1996; individualCount: 1; sex: male; recordedBy: E. Heibo and O. Lønnve; identifiedBy: Andrei E. Humala; institutionCode: ZMUN

f. order: Hymenoptera; family: Ichneumonidae; genus: Cylloceria; specificEpithet: caligata; scientificNameAuthorship: (Gravenhorst, 1829); country: Norway; stateProvince: Akershus; verbatimLocality: Ullensaker, Sessvollmoen, Aurtjernet W.; samplingProtocol: Malaise trap; eventDate: 26.VI-25.VIII.2008; individualCount: 12; sex: males; recordedBy: L.O. Hansen; identifiedBy: Andrei E. Humala; institutionCode: ZMUN

g. order: Hymenoptera; family: Ichneumonidae; genus: Cylloceria; specificEpithet: caligata; scientificNameAuthorship: (Gravenhorst, 1829); country: Norway; stateProvince: Akershus; verbatimLocality: Ullensaker, Sessvollmoen, Aurtjernet W.; samplingProtocol: Malaise trap; eventDate: 26.VI-25.VIII.2008; individualCount: 22; sex: females; recordedBy: L.O. Hansen; identifiedBy: Andrei E. Humala; institutionCode: ZMUN

h. order: Hymenoptera; family: Ichneumonidae; genus: Cylloceria; specificEpithet: caligata; scientificNameAuthorship: (Gravenhorst, 1829); country: Norway; stateProvince: Akershus; verbatimLocality: Ullensaker, Sessvollmoen, Aurtjernet W.; samplingProtocol: Malaise trap; eventDate: 30.VIII-1.X.2008; individualCount: 3; sex: females; recordedBy: L.O. Hansen; identifiedBy: Andrei E. Humala; institutionCode: ZMUN

i. order: Hymenoptera; family: Ichneumonidae; genus: Cylloceria; specificEpithet: caligata; scientificNameAuthorship: (Gravenhorst, 1829); country: Norway; stateProvince: Akershus; verbatimLocality: Ullensaker, Sessvollmoen, Aurtjernet W.; samplingProtocol: Malaise trap; eventDate: 30.VIII-1.X.2008; individualCount: 7; sex: males; recordedBy: L.O. Hansen; identifiedBy: Andrei E. Humala; institutionCode: ZMUN

j. order: Hymenoptera; family: Ichneumonidae; genus: Cylloceria; specificEpithet: caligata; scientificNameAuthorship: (Gravenhorst, 1829); country: Norway; stateProvince:

Buskerud; verbatimLocality: Nedre-Eiker, Mjøndalen, Ryggkollen (W); samplingProtocol: Malaise trap; eventDate: 3.VIII-28.IX.2008; individualCount: 9; sex: males; recordedBy: L.O. Hansen; identifiedBy: Andrei E. Humala; institutionCode: ZMUN

k. order: Hymenoptera; family: Ichneumonidae; genus: Cylloceria; specificEpithet: caligata; scientificNameAuthorship: (Gravenhorst, 1829); country: Norway; stateProvince:

Buskerud; verbatimLocality: Nedre-Eiker, Mjøndalen, Ryggkollen (W); samplingProtocol: Malaise trap; eventDate: 3.VIII-28.IX.2008; individualCount: 5; sex: females; recordedBy: L.O. Hansen; identifiedBy: Andrei E. Humala; institutionCode: ZMUN

Distribution: Holarctic; Finland, Sweden, Lithuania and NW Russia (Karelia).

Notes: This species was reported for Norway several times (Ulbricht 1912, Strand 1913, Roman 1942, Riedel and Berg 1997) but it was synonymised under Cylloceria melancholica Gravenhorst, 1820 without sufficient reasons (Rossem van 1987). According to Humala (2002)C. caligata Gravenhorst is a distinct species, and it should be included again in the list of Norwegian Ichneumonidae. 


\section{Subfamily Diplazontinae Viereck, 1918}

\section{Diplazon sp.1 Klopfstein, 2014 (in press)}

\section{Material}

a. $\quad$ order: Hymenoptera; family: Ichneumonidae; genus: Diplazon; specificEpithet: sp.1; country: Norway; stateProvince: Nord-Trøndelag; verbatimLocality: Høylandet, Skiftesåa stream; eventDate: 1.VII.1986; individualCount: 1; sex: male; recordedBy: John O. Solem; identifiedBy: Seraina Klopfstein; institutionCode: NTNU

Distribution: Western Palaearctic; Norway and Sweden.

Notes: The description of this new species will be published soon (Klopfstein 2014 in press).

\section{Homotropus melanogaster (Holmgren, 1872)}

\section{Material}

a. order: Hymenoptera; family: Ichneumonidae; genus: Homotropus; specificEpithet: melanogaster; scientificNameAuthorship: (Holmgren, 1872); country: Norway; stateProvince: Nordland; verbatimLocality: Vefsn, Røsvaud; individualCount: 1; sex: female; recordedBy: Strand; identifiedBy: Seraina Klopfstein; institutionCode: ZMUN

Distribution: Holarctic; Sweden.

\section{Sussaba hinzi Diller, 1982}

\section{Material}

a. order: Hymenoptera; family: Ichneumonidae; genus: Sussaba; specificEpithet: hinzi; scientificNameAuthorship: Diller, 1982; country: Norway; stateProvince: Oppland; verbatimLocality: Øystre Slidre, Heimdalen; eventDate: 2-3.VIII.1995; individualCount: 1; sex: female; recordedBy: K. Sand; identifiedBy: Seraina Klopfstein; institutionCode: ZMUN

Distribution: Palaearctic; Sweden, NW Russia (Leningrad region).

\section{Syrphoctonus idari Diller, 1985}

\section{Material}

a. order: Hymenoptera; family: Ichneumonidae; genus: Syrphoctonus; specificEpithet: idari; scientificNameAuthorship: Diller, 1985; country: Norway; stateProvince: Nord-Trøndelag; verbatimLocality: Høylandet, Tverråa stream; samplingProtocol: Malaise trap; eventDate: 25.VI.1986; individualCount: 1; sex: female; recordedBy: John O. Solem; identificationID: Seraina Klopfstein; institutionCode: NTNU

Distribution: Western Palaearctic; Sweden. 


\section{Syrphophilus asperatus Dash, 1964}

\section{Materials}

a. order: Hymenoptera; family: Ichneumonidae; genus: Syrphophilus; specificEpithet: asperatus; scientificNameAuthorship: Dash, 1964; country: Norway; stateProvince: Akerhus; verbatimLocality: Ullensaker, Sessvollmoen W; verbatimElevation: 204 m; samplingProtocol: Malaise trap; eventDate: 11-26.VI.2007; habitat: sandy pine forest; individualCount: 1; sex: female; recordedBy: L.O. Hansen; identifiedBy: Seraina Klopfstein; institutionCode: ZMUN

b. order: Hymenoptera; family: Ichneumonidae; genus: Syrphophilus; specificEpithet: asperatus; scientificNameAuthorship: Dash, 1964; country: Norway; stateProvince: Nordland; verbatimLocality: Saltdal, Junkerdalen; samplingProtocol: Malaise trap; eventDate: 31.V-29.VI.2002; habitat: sandy pine forest; individualCount: 1; sex: female; recordedBy: E. Heibo \& O. Lønnve; identifiedBy: Seraina Klopfstein; institutionCode: ZMUN

Distribution: Holarctic; Sweden.

\section{Woldstedtius holarcticus (Diller, 1969)}

Material

a. order: Hymenoptera; family: Ichneumonidae; genus: Woldstedtius; specificEpithet: holarcticus; scientificNameAuthorship: (Diller, 1969); country: Norway; stateProvince: Hedmark; verbatimLocality: Elverum, Starmoen NR (N); samplingProtocol: Malaise trap; eventDate: 11.VI -29.VII.2004; individualCount: 1; sex: female; recordedBy: L.O. Hansen \& E. Rindal; identificationID: Seraina Klopfstein; institutionCode: ZMUN

Distribution: Holarctic and Oriental; Finland.

\section{Subfamily Ichneumoninae Latreille, 1802}

\section{Tribe Ichneumonini Latreille, 1802}

\section{Patrocloides diasemae (Tischbein, 1877)}

\section{Materials}

a. order: Hymenoptera; family: Ichneumonidae; genus: Patrocloides; specificEpithet: diasemae; scientificNameAuthorship: (Tischbein, 1877); country: Norway; stateProvince: Sør-Trøndelag; verbatimLocality: Trondheim: Gjeddvatnet; samplingProtocol: Malaise trap 4; eventDate: 6.VI.2010; individualCount: 2; sex: females; recordedBy: Jon Kr. Skei; identifiedBy: Andrei E. Humala; institutionCode: NTNU

b. order: Hymenoptera; family: Ichneumonidae; genus: Patrocloides; specificEpithet: diasemae; scientificNameAuthorship: (Tischbein, 1877); country: Norway; stateProvince: Sør-Trøndelag; verbatimLocality: Kjerringtjønna; samplingProtocol: Malaise trap 1; eventDate: 6.VI.2010; individualCount: 1; sex: female; recordedBy: Jon Kr. Skei; identifiedBy: Andrei E. Humala; institutionCode: NTNU 
Distribution: Palaearctic; Finland, Sweden and NW Russia (Karelia).

\section{Subfamily Metopiinae Förster, 1869}

\section{Exochus ferus Tolkanitz, 1993}

\section{Material}

a. order: Hymenoptera; family: Ichneumonidae; genus: Exochus; specificEpithet: ferus; scientificNameAuthorship: Tolkanitz, 1993; country: Norway; stateProvince: SørTrøndelag; verbatimLocality: Åfjord, Skjerva Nature Reserve; verbatimElevation: 42 m; verbatimLatitude: $63^{\circ} 56^{\prime} 36.6^{\prime \prime} \mathrm{N}$; verbatimLongitude: $10^{\circ} 16^{\prime 2} 21.0^{\prime \prime} \mathrm{E}$; samplingProtocol: Malaise trap; eventDate: 9.VII-03.IX.2011; habitat: birch forest; individualCount: 1; sex: male; recordedBy: Andrei E. Humala \& Alexey Reshchikov; identifiedBy: Andrei E. Humala; institutionCode: ZMUN

Distribution: Palaearctic.

Notes: New to Scandinavia.

\section{Exochus kuslitzkyi Tolkanitz, 2003}

\section{Materials}

a. order: Hymenoptera; family: Ichneumonidae; genus: Exochus; specificEpithet: kuslitzkyi; scientificNameAuthorship: Tolkanitz, 2003; country: Norway; stateProvince: Hordaland; verbatimLocality: Stord, Leirvik, Gullberg; verbatimElevation: $82 \mathrm{~m}$; verbatimLatitude: $59^{\circ}$ 46'22.4" N; verbatimLongitude: 05³0'42.0" E; samplingProtocol: Malaise trap; eventDate: 6.VII-28.VIII.2011; habitat: broad-leaved forest; individualCount: 1; sex: male; recordedBy: Andrei E. Humala \& Alexey Reshchikov; associatedSequences: voucher for DNA barcoding AH074; identifiedBy: Andrei E. Humala; institutionCode: ZMUN

b. order: Hymenoptera; family: Ichneumonidae; genus: Exochus; specificEpithet: kuslitzkyi; scientificNameAuthorship: Tolkanitz, 2003; country: Norway; stateProvince: Vest-Agder; verbatimLocality: Farsund, Listeid Nature Reserve; verbatimElevation: 50-100 m; verbatimLatitude: $58^{\circ} 07^{\prime} 55^{\prime \prime} \mathrm{N}$; verbatimLongitude: $06^{\circ} 42^{\prime} 57^{\prime \prime} \mathrm{E}$; samplingProtocol: netting; eventDate: 1.VII.2011; habitat: oak forest; individualCount: 1; sex: male; recordedBy: Alexey Reshchikov; identifiedBy: Andrei E. Humala; institutionCode: ZMUN

c. order: Hymenoptera; family: Ichneumonidae; genus: Exochus; specificEpithet: kuslitzkyi; scientificNameAuthorship: Tolkanitz, 2003; country: Norway; stateProvince: Rogaland; verbatimLocality: Tysvær, Nedstrand, Leirå, Tunganeset; verbatimLatitude: 59²0'36" N; verbatimLongitude: $05^{\circ} 52^{\prime} 35 " \mathrm{E}$; samplingProtocol: netting; eventDate: 5.VII.2011; habitat: oak forest; individualCount: 1; sex: male; recordedBy: Alexey Reshchikov; identifiedBy: Andrei E. Humala; institutionCode: ZMUN

Distribution: Palaearctic.

Notes: New to Scandinavia. 


\section{Exochus lictor Haliday, 1839}

\section{Material}

a. order: Hymenoptera; family: Ichneumonidae; genus: Exochus; specificEpithet: lictor, scientificNameAuthorship: Haliday, 1839; country: Norway; stateProvince: Vest-Agder; verbatimLocality: Farsund, Listeid Nature Reserve; verbatimElevation: 133 m; verbatimLatitude: $58^{\circ} 08^{\prime} 35.2^{\prime \prime} \mathrm{N}$; verbatimLongitude: $06^{\circ} 44^{\prime} 11.3^{\prime \prime} \mathrm{E}$; samplingProtocol: Malaise trap; eventDate: 1.VII-24.VIII.2011; habitat: oak forest; individualCount: 1; sex: male; recordedBy: Andrei E. Humala \& Alexey Reshchikov; identifiedBy: Andrei E. Humala; institutionCode: ZMUN

Distribution: Holarctic; Finland, Latvia, Lithuania and NW Russia (Karelia).

\section{Exochus lineifrons Thomson, 1887}

\section{Material}

a. order: Hymenoptera; family: Ichneumonidae; genus: Exochus; specificEpithet: lineifrons; scientificNameAuthorship: Thomson, 1887; country: Norway; stateProvince: Rogaland; verbatimLocality: Tysvær, Silgjerd, Åsen; verbatimElevation: $50 \mathrm{~m}$; verbatimLatitude: $59^{\circ}$ 20'08.6" N; verbatimLongitude: 05³'37.6" E; samplingProtocol: Malaise trap; eventDate: 5.VII-27.VIII.2011; habitat: oak forest; individualCount: 2; sex: females; recordedBy: Andrei E. Humala \& Alexey Reshchikov; associatedSequences: voucher for DNA barcoding AH016; identifiedBy: Andrei E. Humala; institutionCode: ZMUN

Distribution: Palaearctic; Finland and Sweden.

\section{Triclistus globulipes (Desvignes, 1856)}

\section{Material}

a. order: Hymenoptera; family: Ichneumonidae; genus: Triclistus; specificEpithet: globulipes; scientificNameAuthorship: (Desvignes, 1856); country: Norway; stateProvince: AustAgder; verbatimLocality: Lillesand, Vestre Grimevann lake; verbatimLatitude: 58¹9'23.1" $\mathrm{N}$; verbatimLongitude: $08^{\circ} 20^{\prime} 09.1 " \mathrm{E}$; samplingProtocol: Malaise trap; eventDate: 29.VI-23.VIII.2011; habitat: oak forest; individualCount: 1; sex: female; recordedBy: Andrei E. Humala \& Alexey Reshchikov; identifiedBy: Andrei E. Humala; institutionCode: ZMUN

Distribution: Palaearctic and Oriental; Denmark, Finland, Latvia, Lithuania, Sweden and NW Russia (Karelia). 


\title{
Subfamily Pimplinae Wesmael, 1845
}

\author{
Tribe Delomeristini Hellén, 1915
}

\section{Delomerista longicauda Kasparyan, 1973}

\section{Material}

a. order: Hymenoptera; family: Ichneumonidae; genus: Delomerista; specificEpithet: longicauda; scientificNameAuthorship: Kasparyan, 1973; country: Norway; stateProvince: Nord-Trøndelag; verbatimLocality: Høylandet, Tverråa; verbatimElevation: 340 m; samplingProtocol: Malaise trap; eventDate: 30.VII.1986; individualCount: 1; sex: female; recordedBy: Kaare Aagaard; identifiedBy: Andrei E. Humala; institutionCode: NTNU

Distribution: Palaearctic; Sweden.

\section{Tribe Ephialtini Hellén, 1915}

\section{Schizopyga pictifrons (Thomson, 1877)}

\section{Materials}

a. order: Hymenoptera; family: Ichneumonidae; genus: Schizopyga; specificEpithet: pictifrons; scientificNameAuthorship: (Thomson, 1877); country: Norway; stateProvince: Sør-Trøndelag; verbatimLocality: Åfjord, Skjerva Nature Reserve; verbatimElevation: 42 m; verbatimLatitude: $63^{\circ} 56^{\prime} 36.6$ " N; verbatimLongitude: $10^{\circ} 16^{\prime 2} 21.0^{\prime \prime} \mathrm{E}$; samplingProtocol: Malaise trap; eventDate: 09.VII-03.IX.2011; habitat: birch forest; individualCount: 1; sex: male; recordedBy: Andrei E. Humala \& Alexey Reshchikov; identifiedBy: Andrei E. Humala; institutionCode: ZMUN

b. order: Hymenoptera; family: Ichneumonidae; genus: Schizopyga; specificEpithet: pictifrons; scientificNameAuthorship: (Thomson, 1877); country: Norway; stateProvince: Vest-Agder; verbatimLocality: Farsund, Listeid Nature Reserve; verbatimElevation: 50-150 m; verbatimLatitude: 5807'55" N; verbatimLongitude: $06^{\circ} 42^{\prime} 57^{\prime \prime} \mathrm{E}$; samplingProtocol: netting; eventDate: 01.VII.2011; habitat: oak forest; individualCount: 1; sex: male; recordedBy: Alexey Reshchikov; identifiedBy: Andrei E. Humala; institutionCode: ZMUN

Distribution: Palaearctic; Finland and Sweden.

\section{Tribe Pimplini Wesmael, 1845}

\section{Itoplectis insignis Perkins, 1957}

\section{Material}

a. order: Hymenoptera; family: Ichneumonidae; genus: Itoplectis; specificEpithet: insignis; scientificNameAuthorship: Perkins, 1957; country: Norway; stateProvince: Sogn og Fjordane; verbatimLocality: Leikanger, Skopargilet, Bargarden Nature Reserve; verbatimElevation: $114 \mathrm{~m}$; verbatimLatitude: $61^{\circ} 12^{\prime} 18.7^{\prime \prime} \mathrm{N}$; verbatimLongitude: $06^{\circ}$ 
40'30.4" E; samplingProtocol: Malaise trap; eventDate: 7.VII-30.VIII.2011; habitat: broadleaved forest; individualCount: 1; sex: female; recordedBy: A. Humala \& A. Reshchikov; identifiedBy: Alexey Reshchikov; institutionCode: ZMUN

Distribution: Palaearctic; Finland and Sweden.

\section{Subfamily Poemeniinae Narayanan \& Lal, 1953}

\section{Neoxorides montanus Oehlke, 1966}

\section{Materials}

a. order: Hymenoptera; family: Ichneumonidae; genus: Neoxorides; specificEpithet: montanus; scientificNameAuthorship: Oehlke, 1966; country: Norway; stateProvince: Nord-Trøndelag; verbatimLocality: Høylandet, Skiftesåa; Bred inn; verbatimElevation: 200 m; samplingProtocol: Malaise trap; eventDate: 25.VI.1986; individualCount: 1; sex: female; recordedBy: Kaare Aagaard; identifiedBy: Andrei E. Humala; institutionCode: NTNU

b. order: Hymenoptera; family: Ichneumonidae; genus: Neoxorides; specificEpithet: montanus; scientificNameAuthorship: Oehlke, 1966; country: Norway; stateProvince: Nord-Trøndelag; verbatimLocality: Høylandet, Skiftesåa; Bred inn; verbatimElevation: 200 m; samplingProtocol: Malaise trap; eventDate: 15.VII.1987; individualCount: 1; sex: female; recordedBy: Kaare Aagaard; identifiedBy: Andrei E. Humala; institutionCode: NTNU

Distribution: Palaearctic; Finland, NW Russia (Leningrad and Murmansk regions).

\section{Podoschistus scutellaris (Desvignes, 1856)}

\section{Material}

a. order: Hymenoptera; family: Ichneumonidae; genus: Podoschistus; specificEpithet: scutellaris; scientificNameAuthorship: (Desvignes, 1856); country: Norway; stateProvince: Aust-Agder; verbatimLocality: Lillesand, Vestre Grimevann; verbatimElevation: 78 m; verbatimLatitude: $58^{\circ} 19^{\prime 2} 23.1 " \mathrm{~N}$; verbatimLongitude: $08^{\circ} 20^{\prime} 9.1$ " E; samplingProtocol: Malaise trap; eventDate: 29.VI-23.VIII.2011; habitat: oak forest; individualCount: 1; sex: female; recordedBy: A. Humala \& A. Reshchikov; associatedSequences: voucher for DNA barcoding AVR064; identifiedBy: Alexey Reshchikov; institutionCode: ZMUN

Distribution: Palaearctic and Oriental; Sweden and NW Russia.

\section{Subfamily Tersilochinae Schmiedeknecht, 1910}

\section{Diaparsis (Nanodiaparsis) aperta (Thomson, 1889)}

\section{Material}

a. order: Hymenoptera; family: Ichneumonidae; genus: Diaparsis; subgenus: Nanodiaparsis; specificEpithet: aperta; scientificNameAuthorship: (Thomson, 1889); country: Norway; stateProvince: Akerhus; verbatimLocality: Oslo, Bygdøy, Rodeløkken; samplingProtocol: 
Malaise trap; eventDate: 27.VI-25.VII.2006; individualCount: 2; sex: females; recordedBy: A. EndrestøI and S. Olberg; identifiedBy: Andrey Khalaim; institutionCode: ZMUN

Distribution: Palaearctic; Sweden and Finland.

\section{Diaparsis (Diaparsis) carinifer (Thomson, 1889)}

\section{Material}

a. order: Hymenoptera; family: Ichneumonidae; genus: Diaparsis; subgenus: Diaparsis; specificEpithet: carinifer, scientificNameAuthorship: (Thomson, 1889); country: Norway; stateProvince: Akerhus; verbatimLocality: Oslo, Lindøya; samplingProtocol: Malaise trap; eventDate: 19.VI-20.VII.2006; individualCount: 1; sex: female; recordedBy: A. Endrestøl; identifiedBy: Andrey Khalaim; institutionCode: ZMUN

Distribution: Palaearctic; Sweden, Finland and Lithuania.

\section{Diaparsis (Diaparsis) jucunda (Holmgren, 1860)}

\section{Materials}

a. order: Hymenoptera; family: Ichneumonidae; genus: Diaparsis; subgenus: Diaparsis; specificEpithet: jucunda; scientificNameAuthorship: (Holmgren, 1860); country: Norway; stateProvince: Sogn og Fjordane; verbatimLocality: Leikanger, Kvinnefossen waterfall; verbatimElevation: $60 \mathrm{~m}$; verbatimLatitude: $61^{\circ} 12^{\prime} 30.2^{\prime \prime} \mathrm{N}$; verbatimLongitude: $06^{\circ}$ 38'52.0" E; samplingProtocol: Malaise trap; eventDate: 6.VII-30.VIII.2011; habitat: spruce forest with juniper; individualCount: 2; sex: females; recordedBy: Andrei E. Humala \& Alexey Reshchikov; identifiedBy: Andrey Khalaim; institutionCode: ZMUN

b. order: Hymenoptera; family: Ichneumonidae; genus: Diaparsis; subgenus: Diaparsis; specificEpithet: jucunda; scientificNameAuthorship: (Holmgren, 1860); country: Norway; stateProvince: Sogn og Fjordane; verbatimLocality: Leikanger, Skopargilet, Bargarden Nature Reserve; verbatimElevation: 114 m; verbatimLatitude: 61¹2'18.7" N; verbatimLongitude: $06^{\circ} 40^{\prime} 30.4^{\prime \prime} \mathrm{E}$; samplingProtocol: Malaise trap; eventDate: 7.VII-30.VIII.2011; habitat: broad-leaved forest; individualCount: 2; sex: females; recordedBy: Andrei E. Humala \& Alexey Reshchikov; associatedSequences: voucher for DNA barcoding AH060; identifiedBy: Andrey Khalaim; institutionCode: ZMUN

Distribution: Palaearctic; Denmark, Finland, Sweden, and NW Russia (Karelia, Leningrad region).

\section{Epistathmus crassicornis Horstmann, 1971}

\section{Materials}

a. order: Hymenoptera; family: Ichneumonidae; genus: Epistathmus; specificEpithet: crassicornis; scientificNameAuthorship: Horstmann, 1971; country: Norway; stateProvince: Akerhus; verbatimLocality: Follo, Ås, Rustadmåsan; verbatimLatitude: $59^{\circ}$ 39'48.4" N; verbatimLongitude: 1049'15.9" E; samplingProtocol: Malaise trap; eventDate: 15.VII-16.VIII.2011; individualCount: 3; sex: 2 females, 1 male; recordedBy: S.A. Jenseg; identifiedBy: A. Khalaim; institutionCode: ZMUN 
b. order: Hymenoptera; family: Ichneumonidae; genus: Epistathmus; specificEpithet: crassicornis; scientificNameAuthorship: Horstmann, 1971; country: Norway; stateProvince: Hordaland; verbatimLocality: Os, Lyse kloster; verbatimLatitude: $60^{\circ}$ $13^{\prime} 50.8^{\prime \prime} \mathrm{N}$; verbatimLongitude: $05^{\circ} 24^{\prime} 6.9^{\prime \prime} \mathrm{E}$; samplingProtocol: sweeping net; eventDate: 29.VIII.2011; individualCount: 1; sex: male; recordedBy: Alexey Reshchikov; identifiedBy: A. Khalaim; institutionCode: ZMUN

c. order: Hymenoptera; family: Ichneumonidae; genus: Epistathmus; specificEpithet: crassicornis; scientificNameAuthorship: Horstmann, 1971; country: Norway; stateProvince: Sogn og Fjordane; verbatimLocality: Leikanger, Kvinnefossen waterfall; verbatimElevation: $60 \mathrm{~m}$; verbatimLatitude: $61^{\circ} 12^{\prime} 30.2^{\prime \prime} \mathrm{N}$; verbatimLongitude: 6³8'52.0" E; samplingProtocol: Malaise trap; eventDate: 6.VII-30.VIII.2011; habitat: spruce forest with juniper; individualCount: 1; sex: female; recordedBy: A. Humala \& A. Reshchikov; identifiedBy: A.Khalaim; institutionCode: ZMUN

d. order: Hymenoptera; family: Ichneumonidae; genus: Epistathmus; specificEpithet: crassicornis; scientificNameAuthorship: Horstmann, 1971; country: Norway; stateProvince: Sogn og Fjordane; verbatimLocality: Leikanger, Skopargilet; verbatimElevation: $114 \mathrm{~m}$; verbatimLatitude: $61^{\circ} 12^{\prime} 18.7^{\prime \prime} \mathrm{N}$; verbatimLongitude: $06^{\circ}$ 40'30.4" E; samplingProtocol: Malaise trap; eventDate: 6.VII-30.VIII.2011; habitat: broadleaved forest; individualCount: 1; sex: male; recordedBy: A. Humala \& A. Reshchikov; identifiedBy: A.Khalaim; institutionCode: ZMUN

e. order: Hymenoptera; family: Ichneumonidae; genus: Epistathmus; specificEpithet: crassicornis; scientificNameAuthorship: Horstmann, 1971; country: Norway; stateProvince: Vest-Agder; verbatimLocality: Farsund, Listeid Nature Reserve; verbatimElevation: $72 \mathrm{~m}$; verbatimLatitude: $58^{\circ} 08^{\prime} 01.6^{\prime \prime} \mathrm{N}$; verbatimLongitude: $06^{\circ}$ 43'08.2" E; samplingProtocol: Malaise trap; eventDate: 1.VII-24.VIII.2011; habitat: oak forest; individualCount: 3; sex: males; recordedBy: A. Humala \& A. Reshchikov; identifiedBy: A.Khalaim; institutionCode: ZMUN

f. order: Hymenoptera; family: Ichneumonidae; country: Norway; stateProvince: Vest-Agder; verbatimLocality: Farsund, Listeid Nature Reserve; verbatimElevation: 133 m; verbatimLatitude: $58^{\circ} 08^{\prime} 35.2^{\prime \prime} \mathrm{N}$; verbatimLongitude: 06²4'11.3" E; samplingProtocol: Malaise trap; eventDate: 1.VII-24.VIII.2011; habitat: oak forest; individualCount: 2; sex: 1 female, 1 male; recordedBy: A. Humala \& A. Reshchikov; identifiedBy: A.Khalaim; institutionCode: ZMUN

g. order: Hymenoptera; family: Ichneumonidae; country: Norway; stateProvince: Rogaland; verbatimLocality: Tysvær, Silgjerd, Åsen; verbatimElevation: $50 \mathrm{~m}$; verbatimLatitude: $59^{\circ}$ 20'08.6" N; verbatimLongitude: 05²3'37.6" E; samplingProtocol: Malaise trap; eventDate: 5.VII-27.VIII.2011; habitat: oak forest; individualCount: 4; sex: 2 females, 2 males; recordedBy: A. Humala \& A. Reshchikov; identifiedBy: A.Khalaim; institutionCode: ZMUN h. order: Hymenoptera; family: Ichneumonidae; country: Norway; stateProvince: Hordaland; verbatimLocality: Stord, Leirvik, Gullberg; verbatimElevation: $82 \mathrm{~m}$; verbatimLatitude: $59^{\circ}$ 46'22.4" N; verbatimLongitude: $05^{\circ} 30^{\prime} 42.0^{\prime \prime} \mathrm{E}$; samplingProtocol: Malaise trap; eventDate: 6.VII-28.VIII.2011; habitat: broad-leaved forest; individualCount: 2; sex: 1 female, 1 male; recordedBy: A. Humala \& A. Reshchikov; identifiedBy: A.Khalaim; institutionCode: ZMUN

i. $\quad$ order: Hymenoptera; family: Ichneumonidae; country: Norway; stateProvince: Aust-Agder; verbatimLocality: Lillesand, Vestre Grimevann lake; verbatimLatitude: 58¹9'23.1" N; verbatimLongitude: $08^{\circ} 20^{\prime} 09.1$ " E; samplingProtocol: Malaise trap; eventDate: 29.VI-23.VIII.2011; habitat: oak forest; individualCount: 2; sex: 1 female, 1 male; recordedBy: A. Humala \& A. Reshchikov; identifiedBy: A.Khalaim; institutionCode: ZMUN 
j. $\quad$ order: Hymenoptera; family: Ichneumonidae; country: Norway; stateProvince: SørTrøndelag; verbatimLocality: Åfjord, Skjerva Nature Reserve; verbatimElevation: 42 m; verbatimLatitude: 6356'36.6" N; verbatimLongitude: 10¹6'21.0" E; samplingProtocol: Malaise trap; eventDate: 9.VII-3.IX.2011; habitat: birch forest; individualCount: 1; sex: male; recordedBy: A. Humala \& A. Reshchikov; identifiedBy: A.Khalaim; institutionCode: ZMUN

Distribution: Palaearctic; Denmark, Finland and NW Russia (Karelia).

\section{Gelanes simillimus Horstmann, 1981}

\section{Materials}

a. order: Hymenoptera; family: Ichneumonidae; genus: Gelanes; specificEpithet: simillimus; scientificNameAuthorship: Horstmann, 1981; country: Norway; stateProvince: Akerhus; verbatimLocality: Follo, Ås, Åsmåsan; verbatimElevation: 126 m; verbatimLatitude: 59 40'16.7" N; verbatimLongitude: $10^{\circ} 46$ '53.9" E; samplingProtocol: Malaise trap; eventDate: 16-19.V.2011; individualCount: 2; sex: 1 female, 1 male; recordedBy: S.A. Jenseg; identifiedBy: A.Khalaim; institutionCode: ZMUN

b. $\quad$ order: Hymenoptera; family: Ichneumonidae; genus: Gelanes; specificEpithet: simillimus; scientificNameAuthorship: Horstmann, 1981; country: Norway; stateProvince: Akerhus; verbatimLocality: Follo, Ås, Rustadmåsan; verbatimLatitude: $59^{\circ} 39^{\prime} 48.4^{\prime \prime} \mathrm{N}$; verbatimLongitude: $10^{\circ} 49^{\prime} 15.9^{\prime \prime} \mathrm{E}$; samplingProtocol: Malaise trap; eventDate: 15.VII-16.VIII.2011; individualCount: 2; sex: 1 female, 1 male; recordedBy: S.A. Jenseg; identifiedBy: A.Khalaim; institutionCode: ZMUN

Distribution: Palaearctic; Sweden, Finland, Lithuania and NW Russia.

\section{Probles (Euporizon) extensor (Aubert, 1971)}

\section{Material}

a. order: Hymenoptera; family: Ichneumonidae; genus: Probles; subgenus: Euporizon; specificEpithet: extensor, scientificNameAuthorship: (Aubert, 1971); country: Norway; stateProvince: Oppland; verbatimLocality: Slidre, Heimdalen; samplingProtocol: Malaise trap; eventDate: 2-3.VIII.1995; individualCount: 1; sex: female; recordedBy: K. Sand; identifiedBy: A.Khalaim; institutionCode: ZMUN

Distribution: Western Palaearctic.

Notes: New to Scandinavia.

\section{Tersilochus (Tersilochus) longicornis (Thomson, 1889)}

\section{Material}

a. order: Hymenoptera; family: Ichneumonidae; genus: Tersilochus; subgenus: Tersilochus; specificEpithet: Iongicornis; scientificNameAuthorship: (Thomson, 1889); country: Norway; stateProvince: Aust-Agder; verbatimLocality: Birkenes, Birkeland; samplingProtocol: Malaise trap; eventDate: V.2006; individualCount: 3; sex: females; recordedBy: Svein Svendsen; identifiedBy: Andrey Khalaim; institutionCode: ZMUN 
Distribution: Palaearctic; Sweden, Finland and NW Russia (Leningrad region).

\section{Tersilochus (Tersilochus) triangularis Gravenhorst, 1807)}

Material

a. order: Hymenoptera; family: Ichneumonidae; genus: Tersilochus; subgenus: Tersilochus; specificEpithet: triangularis; scientificNameAuthorship: (Gravenhorst, 1807); country: Norway; stateProvince: Akerhus; verbatimLocality: Oslo, Ekebergskråningen [NW]; samplingProtocol: Malaise trap; eventDate: 24.IV-29.V.2007; individualCount: 1; sex: female; recordedBy: A. Endrestøl and L.O. Hansen; identifiedBy: Andrey Khalaim; institutionCode: ZMUN

Distribution: Palaearctic; Sweden, Finland and Lithuania.

\section{Subfamily Tryphoninae Shuckard, 1840}

\section{Tribe Exenterini Förster, 1869}

\section{Eridolius clauseni (Kerrich, 1962)}

Material

a. order: Hymenoptera; family: Ichneumonidae; genus: Eridolius; specificEpithet: clauseni; scientificNameAuthorship: (Kerrich, 1962); country: Norway; stateProvince: Akerhus; verbatimLocality: Baerum, Oksenøya, Oksenøyveinen no.71; samplingProtocol: Malaise trap; eventDate: 1.VII-15.VIII.2003; individualCount: 1; sex: female; recordedBy: L.O. Hansen; identifiedBy: Alexey Reshchikov; institutionCode: ZMUN

Distribution: Palaearctic and Oriental.

Notes: New to Scandinavia. In Western Palaearctic reported from Germany only.

\section{Eridolius deletus (Thomson, 1883)}

Material

a. order: Hymenoptera; family: Ichneumonidae; genus: Eridolius; specificEpithet: deletus; scientificNameAuthorship: (Thomson, 1883); country: Norway; stateProvince: Oppland; verbatimLocality: Sør-Aurdal, Kjenstrud; eventDate: VII.1995; individualCount: 1; sex: female; recordedBy: Garli; identifiedBy: Alexey Reshchikov; institutionCode: ZMUN

Distribution: Palaearctic; Sweden, Finland and NW Russia (Karelia). 


\section{Exenterus confusus Kerrich, 1952}

\section{Material}

a. order: Hymenoptera; family: Ichneumonidae; genus: Exenterus; specificEpithet: confusus; scientificNameAuthorship: Kerrich, 1952; country: Norway; stateProvince: Sør-Trøndelag; verbatimLocality: Åfjord, Skjerva Nature Reserve; verbatimElevation: 42 m; verbatimLatitude: $63^{\circ} 56^{\prime} 36.6^{\prime \prime} \mathrm{N}$; verbatimLongitude: $10^{\circ} 16^{\prime 2} 21.0^{\prime \prime} \mathrm{E}$; samplingProtocol: Malaise trap; eventDate: 9.VII-3.IX.2011; habitat: birch forest; individualCount: 1; sex: female; recordedBy: Andrei E. Humala \& Alexey Reshchikov; identifiedBy: Andrei E. Humala; institutionCode: ZMUN

Distribution: Holarctic; Finland, Sweden and NW Russia (Karelia).

\section{Tribe Tryphonini Shuckard, 1840}

\section{Polyblastus (Polyblastus) cothurnatus (Gravenhorst, 1829)}

\section{Material}

a. order: Hymenoptera; family: Ichneumonidae; genus: Polyblastus; subgenus: Polyblastus; specificEpithet: cothurnatus; scientificNameAuthorship: (Gravenhorst, 1829); country: Norway; stateProvince: Aust-Agder; verbatimLocality: Lillesand, Vestre Grimevann lake; verbatimLatitude: 58¹9'23.1" N; verbatimLongitude: 08²0'09.1" E; samplingProtocol: Malaise trap; eventDate: 29.VI-23.VIII.2011; habitat: oak forest; individualCount: 1; sex: female; recordedBy: Andrei E. Humala \& Alexey Reshchikov; identifiedBy: Andrei E. Humala; institutionCode: ZMUN

Distribution: Palaearctic; Denmark, Finland, Sweden, Latvia, Lithuania and NW Russia (Karelia).

\section{Acknowledgements}

The present study was funded by Artsdatabanken (Artsprosjektet) grant to Dr. Vladimir Gusarov (project No 70184219 "Filling the blanks: a quest for overlooked Norwegian species in poorly known insect taxa: Hymenoptera: Ichneumonidae"). For the loan of Ichneumonidae material, we would like to thank all the curators and other colleagues who generously made the specimens under their care available for this study: Dr. Torbjørn Ekrem (NTNU), Mr. Lars Ove Hansen (ZMUN), Dr. Steffen Roth and Mr. Per Djursvoll (University Museum of Bergen), Ms Sanna Leppänen (UEF), curators of Swedish museums for making possible to examine the type specimens deposited in their collections: Dr. Hege Vårdal (The Swedish Museum of Natural History, Stockholm), Dr. Hans Mejlon (Zoological Institute of Uppsala University), Dr. Roy Danielsson (Zoological Institute, Lund). We are grateful to Dr. Andrey Khalaim (Zoological Institute, Russian Academy of Sciences) for his help in identification of the Tersilochinae specimens, and Dr. Seraina Klopfstein (The University of Adelaide) for her help in identifications of the Diplazontinae specimens. We also are very grateful to Mr. Finn Audun Grøndahl (Lands 
Museum, Dokka) who kindly helped us during the field work in Oppland. We are indebted to Dr. Vladimir Gusarov (ZMUN) for critically reading this manuscript and for useful comments on the text, Dr. Andrey Khalaim and Dr. Dmitri Kasparyan for reviews of manuscript (Zoological Institute, Russian Academy of Sciences). Ms. Synne Arnesen Jenseg (Norwegian University of Life Sciences, Ås), Ms. Dolores Lucía Guzman Herrador (University of Granada, Spain) and Mrs. Lydia Kopilyas (Russia) are thanked for their assistance in field work and material sorting.

\section{References}

- $\quad$ Achterberg van C, Zwakhals K (2004) Fauna Europaea: Ichneumonoidea. 2.4.

URL: http://www.faunaeur.org/

- $\quad$ Broad G (2011) Checklist of British and Irish Ichneumonidae (Hymenoptera). Release date: 20118 05. URL: http://www.nhm.ac.uk/resources-rx/files/ ichneumonidae-checklist-for-web-27752.pdf

- Hansen LO, Humala A, Reschikov AV (2010) Ichneumonidae (Hymenoptera) new for the fauna of Norway, Part 4. Norwegian Journal of Entomology 57: 177-186. [In English].

- Horstmann K (2004) Bemerkungen zur Systematik einiger Gattungen der Campopleginae IV (Hymenoptera, Ichneumonidae). Zeitschrift der Arbeitsgemeinschaft Österreichischer Entomologen 56: 13-35. [In German].

- Humala AE (2002) A review of the Parasitic Wasps of the Genera Cylloceria Schiødte, 1838 and Allomacrus Förster, 1868 (Hymenoptera, Ichneumonidae) of the Fauna of Russia. Entomologicheskoe Obozrenie 82 (3): 301-313. [In English].

- Humala AE, Reshchikov AV (2012) Ichneumonidae (Hymenoptera) new to the Norwegian fauna. Norwegian Journal of Entomology 59: 67-71. [In English].

- Kasparyan DR, Khalaim Al (2007) Ctenopelmatinae. In: Lelej AS (Ed.) Key to the insects of Russia Far East. Volume IV. Neuropteroidea, Mecoptera, Hymenoptera.

4. Dal'nauka, Vladivostok, 474-559 pp. [In Russian].

- Kasparyan DR, Kopelke JP (2009) Taxonomic review and key to European ichneumon-flies (Hymenoptera, Ichneumonidae), parasitoids of gall-forming sawflies of the genera Pontania Costa, Phyllocolpa Benson, and Euura Newman (Hymenoptera, Tenthredinidae) on willows: Part I. Entomological Review 89 (8): 933-957. [In English]. DOI: 10.1134/S0013873809080089

- Kasparyan DR, Kopelke JP (2010) A taxonomic review of ichneumon-flies (Hymenoptera, Ichneumonidae), parasitoids of gall-forming sawflies (Hymenoptera, Tenthredinidae) on Salix. Part II. Review of the Palaearctic species of the genus Saotis Förster with description of four new species. Entomological Review 90 (1): 71-98. [In English]. DOI: 10.1134/S0013873810010069

- Klopfstein S (2014) Revision of the Western Palaearctic Diplazontinae (Hymenoptera, Ichneumonidae). European Journal of Taxonomy 0: 0-0. [In English]. 
- Kopelke JP (1994) Der Schmarotzerkomplex (Brutparasiten und Parasitoide) der gallenbildenden Pontania-Arten (Insecta: Hymenoptera: Tenthredinidae).

Senckenbergiana Biologica 73: 83-133. [In German].

- Koponen M, Jussila R, Vikberg V (1995) A check list of Finnish Hymenoptera, Parasitica, part 1. family Ichneumonidae, subfamilies Pimplinae, Poemeninae, Rhyssinae and Diacritinae. Sahlbergia 2: 87-98. [In Finnish].

- Reshchikov A (2013) Two new species of Lathrolestes (Hymenoptera, Ichneumonidae) from Norway, northern Russia and Finland with a key to western Palaearctic species. Zootaxa 3681 (1): 59. [In English]. DOI: 10.11646/ zootaxa.3681.1.3

- $\quad$ Riedel M, Berg $\varnothing$ (1997) Faunistic Distribution on Norwegian Ichneumonidae (Hymenoptera). Fauna norvegica B 44: 39-53. [In English].

- $\quad$ Riedel M, Hansen LO (2007) Ichneumonidae (Hymenoptera) new for the fauna of Norway. Part 3. Norwegian Journal of Entomology 54: 59-64. [In English].

- Riedel M, Hansen LO (2012) Ichneumonidae (Hymenoptera) new for the fauna of Norway. Part 5. Norwegian Journal of Entomology 59: 219-228. [In English].

- $\quad$ Riedel M, Hansen LO (2013) Ichneumonidae (Hymenoptera) new for the fauna of Norway. Part 6. Norwegian Journal of Entomology 60: 190-195. [In English].

- $\quad$ Riedel M, Hansen LO, Berg $\varnothing$ (2000) Ichneumonidae (Hymenoptera) new for the fauna of Norway. Norwegian Journal of Entomology 47: 163-176. [In English].

- $\quad$ Riedel M, Hansen LO, Berg $\varnothing$ (2005) Ichneumonidae (Hymenoptera) new for the fauna of Norway. Part 2. Norwegian Journal of Entomology 52: 151-162. [In English].

- Roman A (1942) List of new finds of Ichneumonidae in Norway. Tromsø Mus. Årsheft. Nat. Avd. 23 (60): 1-20. [In English].

- Rossem van G (1987) A Revision of Western Palaearctic Oxytorine Genera. Part VI. Tijdschrift voor Entomologie 130: 49-108. [In English].

- Strand E (1913) Hymenoptera Parasitica. Neue Beiträge zur Arthropoden-Fauna Norwegens nebst gelegenlichen Bemerkungen über die deutchen Arten. Nyt Mag. Naturvidensk. 51: 337-361. [In German].

- Ulbricht A (1912) Pimplinae. In Strand, E., Hymenoptera Parasitica. Neue Beiträge zur Arthropoden-Fauna Norwegens nebst gelegenlichen Bemerkungen über die deutchen Arten. VIII. Nyt Mag. Naturvidensk 50: 23-28. [In German].

- Yu DS, van Achterberg K, Horstmann K (2012) Taxapad 2012, Ichneumonoidea 2011. Database on flash-drive. Ottawa, Ontario, Canada. URL: www.taxapad.com 